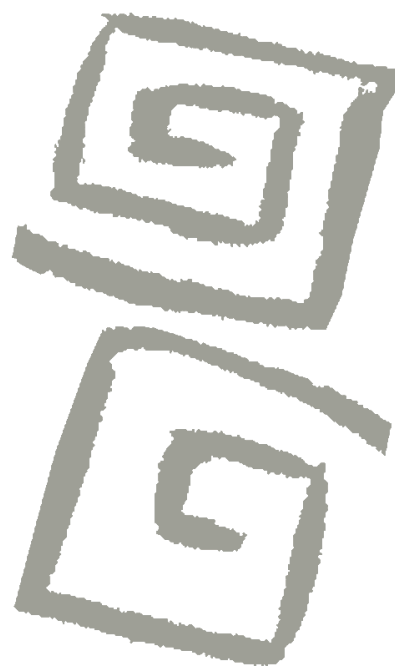

\title{
Análisis de la tendencia e impacto de la mortalidad por causas externas: México, 2000-2013
}

\author{
Analysis of the trend and impact of mortality due to \\ external causes: Mexico, 2000-2013
}

Claudio Alberto Dávila Cervantes ${ }^{1}$, Ana Melisa Pardo Montaño ${ }^{2}$

${ }^{1}$ Doctor en Estudios de Población. ProfesorInvestigador, Facultad Latinoamericana de Ciencias Sociales, México. $\triangle$ iD

2Doctora en Geografía. Profesora-Investigadora Instituto de Geografía, Universidad Nacional Autónoma de México, México. $\square$ (iD
RESUMEN El objetivo de este artículo es analizar la mortalidad por las principales causas externas (accidentes de tránsito, otros accidentes, homicidios y suicidios) en México, calculando los años de vida perdidos entre 0 y 100 años de edad y su contribución al cambio en la esperanza de vida entre 2000 y 2013, a nivel nacional, por sexo y grupos de edad. La información provino de las estadísticas vitales de mortalidad del Instituto Nacional de Estadística y Geografía (INEGI). El mayor impacto de la mortalidad por causas externas entre 2000 y 2013 se presentó en hombres jóvenes y adultos de 15 a 49 años de edad; la mortalidad por estas causas se mantuvo constante en hombres, mientras que disminuyó en mujeres. La mortalidad por accidentes de tránsito y otros accidentes disminuyó, lo que significó un aporte positivo a la esperanza de vida, que fue cancelado por un incremento de la mortalidad por homicidios y suicidios. La mortalidad por causas externas es prevenible por medio de intervenciones, programas y estrategias de prevención y tratamiento oportuno. Es necesario desarrollar estudios multidisciplinarios sobre la dinámica de los factores asociados con la mortalidad por estas causas.

PALABRAS CLAVES Mortalidad; Causas de Muerte; Esperanza de Vida; México.

\begin{abstract}
The objective of this study was to analyze mortality due to the main external causes of death (traffic accidents, other accidents, homicides and suicides) in Mexico, calculating the years of life lost between 0 and 100 years of age and their contribution to the change in life expectancy between 2000 and 2013, at the national level, by sex and age group. Data came from mortality vital statistics of the Instituto Nacional de Estadística y Geografía (INEGI) [National Institute of Statistics and Geography]. The biggest impact in mortality due to external causes occurred in adolescent and adult males 15-49 years of age; mortality due to these causes remained constant in males and slightly decreased in females. Mortality due to traffic accidents and other accidents decreased, with a positive contribution to life expectancy, but this effect was canceled out by the increase in mortality due to homicides and suicides. Mortality due to external causes can be avoided through interventions, programs and prevention strategies as well as timely treatment. It is necessary to develop multidisciplinary studies on the dynamics of the factors associated with mortality due to these causes.
\end{abstract}

KEY WORDS Mortality; Cause of Death; Life Expectancy; Mexico. 


\section{INTRODUCCIÓN}

La mortalidad por causas externas es uno de los problemas de salud pública de mayor importancia en Latinoamérica ${ }^{(1,2)}$ y, además, es un indicador de desigualdades e inequidades en salud que tiene gran impacto social y económico en la población ${ }^{(1)}$. La tasa de mortalidad por causas externas en la región presenta niveles muy elevados y recientemente ha incrementado su efecto en la mortalidad general ${ }^{(1,3)}$. Las causas externas de mortalidad se pueden clasificar en intencionales (suicidio y homicidio), no intencionales (accidentes de tránsito u otros accidentes) y de intención indeterminada ${ }^{(4)}$.

La Organización Mundial de la Salud (OMS) estima que, a nivel mundial, 1,6 miIlones de personas mueren anualmente por actos violentos (520.000 por homicidios y 815.000 por suicidios) cifra que equivale a más del $2,5 \%$ del total de defunciones ${ }^{(5,6,7)}$; por accidentes de tránsito fallecen 1,2 miIlones de personas, lo que equivale al 2,25\% del total de defunciones ${ }^{(8)}$; y por otros accidentes fallecen más de 2,4 millones de personas al año, lo que equivale al $4,41 \%$ del total de fallecimientos ${ }^{(9)}$.

Las defunciones por causas externas afectan a toda la población; sin embargo, el grupo de edad en el que tienen una mayor incidencia es entre los 15 y los 49 años, para el cual es la principal causa de muerte en todo el mundo ${ }^{(1,5,7)}$. Otra característica es que, además de los 5 millones de personas que mueren anualmente por estas causas, un gran número adicional sufre lesiones no fatales producto de estas ${ }^{(5)}$. La OMS estima que todos los días miles de personas son víctimas de este tipo de causas, las cuales resultan con lesiones, muchas de ellas graves, por lo que requieren cuidados médicos, psicológicos o de rehabilitación ${ }^{(8)}$, lo que acarrea costos sociales y costos económicos que alcanzan los miles de millones de dólares ${ }^{(1,5,7)}$.

En México, la participación proporcional de las muertes por causas externas dentro del total de defunciones ha aumentado en los últimos años ${ }^{(8,10,11)}$. Más de 64.000 personas murieron en 2013 por este tipo de causas; en ese mismo año, los homicidios fueron la sexta causa de muerte a nivel nacional; otros accidentes, la octava causa de muerte; los accidentes de tránsito, la décima; y los suicidios ocuparon el décimo quinto lugar ${ }^{(12)}$. Los homicidios han mostrado un incremento en los últimos años, después de un periodo de tendencia decreciente ${ }^{(10,13)}$. De acuerdo con las estadísticas vitales, el número de homicidios registrados entre 2000 y 2007 no presentó grandes variaciones pero, a partir de 2008, se registró un incremento significativo de defunciones por esta causa ${ }^{(12)}$. Cada año mueren en accidentes de tránsito, en promedio, 16.500 mexicanos; pero cabe destacar que, en 2011, las 16.615 personas que fallecieron son un pequeño porcentaje de los 412.087 accidentes de tránsito reportados ${ }^{(8)}$. En cuanto al suicidio, aun cuando su participación dentro de este grupo de causas de muerte ha sido la menor en el país ${ }^{(14)}$, en las últimas cuatro décadas ha tenido un incremento ininterrumpido.

Dado que la mortalidad por causas externas constituye un problema complejo de salud pública y que una aproximación al conocimiento del estado de salud de los mexicanos se da a través del estudio de las causas de muerte ${ }^{(15)}$, el objetivo principal del presente artículo es analizar la carga de mortalidad por las principales causas externas en México, utilizando los años de vida perdidos (AVP), y la contribución de dichas causas de muerte al cambio en la esperanza de vida entre 2000 y 2013, a nivel nacional, por sexo y grupos de edad.

\section{MATERIAL Y MÉTODOS}

La información provino de las estadísticas vitales de mortalidad del Instituto Nacional de Estadística y Geografía (INEGI), para el periodo 2000-2013. Se consideraron fallecimientos por causas externas intencionales (homicidios y suicidios), y no intencionales (accidentes de tránsito y otros accidentes), siguiendo la línea de diversos estudios ${ }^{(1,6,16,17)}$. Se seleccionaron las causas de muerte, bajo 
los criterios de la Clasificación Internacional de Enfermedades (CIE-10), ubicadas en el capítulo "Causas externas de morbilidad y de mortalidad", con códigos: accidentes de tránsito (V00-V99); otros accidentes (W00-X59); homicidios (X85-Y09, Y87.1); y suicidios (X60-X84, Y87.0). Cabe señalar que en este estudio no se tomó en cuenta el apartado "Eventos de intención no determinada" de la CIE-10; sin embargo, con el objetivo de descartar la existencia de posibles sesgos por la subestimación en las causas de muerte consideradas, se analizó la tendencia de los fallecimientos clasificados como lesiones de intención no determinada (Y10-Y34).

Se realizó un prorrateo para distribuir las muertes de edades no especificadas entre el resto de los grupos de edad. Únicamente se tuvieron en cuenta defunciones ocurridas al interior del país. Se excluyeron: 1) muertes ocurridas en el exterior (1.273 registros); 2) registros no especificados por sexo (1.230 casos); 3) para los AVP, las defunciones de mayores de 100 años (55.253 casos en total). A partir de esto, se descartó el $0,76 \%$ del total de registros para el cálculo de los AVP.

Se calcularon los AVP propuestos por Arriaga para estimar cuántos años de vida en promedio se perdieron entre 2000 y 2013 por defunciones por causas externas de personas menores a 100 años de edad. Es decir, se trató de determinar cuántos años más deberían haber vivido las personas que fallecieron por estas causas antes de los 100 años. Se utilizó el supuesto de mortalidad nula entre las dos edades elegidas, por el que se asume que aquellos que mueren deberían haber vivido hasta los 100 años de edad. Este supuesto tiene la ventaja de que, al comparar la mortalidad observada con la mortalidad hipotética nula, las comparaciones quedan estandarizadas ${ }^{(18)}$. El otro supuesto importante del método se basa en que la distribución de las defunciones por causa de muerte de la tabla de mortalidad es igual a la observada en las defunciones registradas. Una posible desventaja de esto es que se limita la edad superior, por lo que se recomienda tomar en todos los casos la edad más alta posible y esta limitación es poco significativa cuando dicha edad es muy avanzada ${ }^{(19)}$ como en esta investigación. Esta técnica es una de las principales herramientas para medir los cambios en el nivel de la mortalidad y facilitar la interpretación y comprensión del estudio de la mortalidad por causas ${ }^{(18,19)}$ dando cuenta de los cambios e impacto que tienen estas causas de muerte en la salud de la población.

Se calcularon tablas de vida para 2000 y 2013, para obtener el cambio en la esperanza de vida entre esos años. Para el cálculo de la contribución de las distintas causas de muerte por grupos de edad al cambio en la esperanza de vida, se utilizó la extensión del modelo de Andrevv ${ }^{(20)}$ realizada en otros estudios $^{(21,22)}$. El cambio en la esperanza de vida $\left(e_{0}\right)$ entre el tiempo 1 (año 2000) y el tiempo 2 (año 2013) se obtuvo como:

$$
e_{0}^{2}-e_{0}^{1}=\sum_{x=0}^{\omega-5} \sum_{j=1}^{k}{ }_{5} C_{x}^{j}
$$

Donde:

${ }_{5} C_{x}^{j}=\frac{1}{2}\left[\frac{{ }_{5} R_{x}^{j, 2} \ln \left\{{ }_{5} p_{x}^{2}\right\}-{ }_{5} R_{x}^{j, 1} \ln \left\{{ }_{5} p_{x}^{1}\right\}}{\ln \left\{{ }_{5} p_{x}^{2}\right\}-\ln \left\{{ }_{5} p_{x}^{1}\right\}}\right]\left[{ }_{5} \delta_{x}\right]$

con

${ }_{5} \delta_{x}=\left\{\left[e_{x}^{2}-e_{x}^{1}\right]\left[{ }_{x} p_{0}^{1}+{ }_{x} p_{0}^{2}\right]-\left[e_{x+5}^{2}-e_{x+5}^{1}\right]\left[{ }_{x+5} p_{0}^{1}+{ }_{x+5} p_{0}^{2}\right]\right\}$

${ }_{5} R_{x}^{j, i}$ es la proporción del total de defunciones atribuidas a una causa de muerte $j$, en el momento $i ;{ }_{n} p_{x}$ es la probabilidad que tiene un superviviente de $x$ años de edad en la tabla de vida de vivir $n$ años más; $e_{x}^{i}$ es la esperanza de vida a los $x$ años de edad, en el momento $i$; y los índices 1 y 2 indican el año inicial y el año final, respectivamente.

Ambos métodos de análisis son complementarios. Con los AVP se tiene un panorama del momento de la mortalidad por causas externas y su tendencia en el periodo de estudio; con el otro método se tiene la 
descomposición de la esperanza de vida temporaria por edad y causas de muerte a lo largo de todo el periodo de estudio(11), lo que proporciona una mirada longitudinal del impacto de la mortalidad por estas causas en México entre 2000 y 2013.

\section{RESULTADOS}

Entre 2000 y 2013 se registraron 7.291.266 defunciones; de las cuales el $3,16 \%$ corresponde a accidentes de tránsito, fue la quinta causa de muerte; otros accidentes, con un $3,78 \%$, fue la sexta causa de muerte; un 2,96\% de las defunciones fueron por homicidios, siendo la novena causa de muerte; y los suicidios ascendieron al 0,88\%, por lo que se ubicaron como la decimonovena causa de muerte. Para hombres, los homicidios representaron un $4,7 \%$ del total de defunciones masculinas en el periodo (4.063.539); los accidentes de tránsito, un $4,5 \%$; los suicidios, un $1,3 \%$; y otros accidentes, representó un 5,1\%. Para mujeres, en cambio, del total de defunciones (3.223.625), los accidentes de tránsito correspondieron al $1,5 \%$; los homicidios, al 0,7\%; los suicidios, al $0,4 \%$; y otros accidentes, al $2,1 \%$, lo que representa una participación relativa menor en el perfil epidemiológico femenino. Dentro del conjunto de causas externas de muerte, los homicidios tuvieron una participación relativamente estable entre 2000 y 2007 (alrededor del $20 \%$ en hombres y del $11,5 \%$ en mujeres); sin embargo, a partir de 2008 y hasta 2013 se incrementó (36\% en hombres y $20,5 \%$ en mujeres); en el caso de los suicidios dicha participación relativa se mantuvo estable para hombres $(8 \%)$ pero, en mujeres, se incrementó de manera paulatina (del 5,3\% en 2000 al 8,4\% en 2013); en el caso de los accidentes de tránsito, su participación relativa hasta 2006 fue creciente $(32 \%$ para hombres y $33,5 \%$ para mujeres) pero, a partir de ese año, disminuye de manera continua $(23,4 \%$ para hombres y $25,5 \%$ para mujeres, en 2013) y, en el caso de otros accidentes, se tuvo una tendencia decreciente en todo el periodo para ambos sexos (del $37 \%$ para hombres y el $46 \%$ para mujeres, en 2000 , al $25 \%$ y al $37 \%$, en 2013 , respectivamente), excepto en 2007, año en el que tuvieron un repunte importante. Por otro lado, los eventos de intención no determinada tuvieron una participación relativamente estable dentro del conjunto de causas externas de muerte (alrededor del $5 \%$ en hombres y del $4 \%$ en mujeres), excepto en 2011, año en el cual se presentó un incremento en ambos sexos ( $7,6 \%$ hombres y $6,2 \%$ en mujeres).

\section{Años de vida perdidos}

Las causas externas de muerte consideradas en este estudio presentaron, en conjunto, en 2000, 4,53 AVP para hombres y 2,59 para mujeres; esto implica que, si se eliminara la mortalidad por esta causa, la esperanza de vida temporaria entre 0 y 100 años se incrementaría 4,53 años para hombres y 2,59 en mujeres. Para 2013, la mortalidad por causas externas para hombres tuvo un ligero incremento del 0,21\% (4,54 años), mientras que para mujeres disminuyó al $10,7 \%$ (2,31 años).

Por causa de muerte, para hombres, en 2000, la mayor cantidad de AVP fue por otros accidentes (1,81 años) y accidentes de tránsito (1,34 años), que presentaron una tendencia decreciente (Figura 1). En 2013, los AVP por otros accidentes alcanzaron 1,22 años $y$, por accidentes de tránsito, 1,16 años, con un descenso del $32,7 \%$ y del $13,3 \%$ respectivamente. Cabe destacar que para otros accidentes, el $48 \%$ del total de AVP se presenta entre los 15 y los 49 años de edad mientras que, para los accidentes de tránsito, este porcentaje es mayor al $66 \%$. La situación es distinta para homicidios, ya que en 2000 se perdieron 1,05 años de vida y, hasta 2007, la tendencia fue decreciente (alcanzando 0,74 años). Sin embargo, a partir de 2008, se produjo un incremento significativo de la mortalidad por homicidios, que alcanzó un máximo de 2,1 AVP en 2011, y disminuyó posteriormente hasta 1,74 años, lo que ubica actualmente a los homicidios masculinos como la principal 
causa de muerte violenta en México. El impacto de la mortalidad por homicidios masculinos se concentra entre los 15 y los 49 años de edad, y alcanza el $82,7 \%$ del total de AVP por esta causa. Los suicidios tuvieron los menores AVP en todo el periodo para hombres (0,33 años, en 2000, y 0,42 años, en 2013); pero muestran una tendencia creciente a lo largo de todo el periodo de estudio, con un aumento del $34,1 \%$ que sostiene el incremento observado desde la década de 1970, reportado en otras investigaciones. El mayor impacto de los suicidios se presenta entre los 15 y los 49 años de edad con un $76 \%$ del total de AVP por esta causa. Llama la atención que los AVP por lesiones de intención no determinada tuvieron una tendencia relativamente constante a lo largo del periodo (alrededor del 0,25 años) excepto en 2011, cuando presentan un incremento importante respecto de años anteriores (0,38 años).

Para mujeres, en 2000, la mayor cantidad de AVP se dio por otros accidentes $(1,15$ años) y accidentes de tránsito (0,75 años) y, al igual que para hombres, con una tendencia decreciente, hasta ubicarse en 0,76 años para otros accidentes y 0,59 años en accidentes de tránsito (con un descenso del $33,5 \%$ y el $20,8 \%$, respectivamente) (Figura 2). Resalta que, para otros accidentes, el grupo de edad con el mayor número de AVP fue el de 65 a 84 años, con un $26,1 \%$ en 2013, seguido por el de 50 a 64 años $(19,5 \%)$ y el de 30 a 49 años $(19,2 \%)$. Para los accidentes de tránsito la mayoría de los AVP ocurren entre los 15 y los 84 años de edad, pero se distribuyen uniformemente entre esas edades $(21,6 \%$ en el grupo $15-29$ años, $26,5 \%$ en $30-49$ años, 20,2\% en 50-64 años y $19 \%$ en 65-84 años). Para homicidios femeninos, en 2000, se perdieron 1,05 años de vida con una tendencia decreciente hasta 2007 (0,38 AVP). Pero, a partir de 2008, se presenta un incremento de la mortalidad por homicidios que alcanza un máximo de 0,87 AVP en 2011, y disminuye hasta 0,76 AVP en 2013 (similar a otros accidentes). La mayor mortalidad por homicidios femeninos se presenta entre los 15 y los 49 años de edad, con $70 \%$ de los AVP por esta causa. Finalmente, los suicidios femeninos

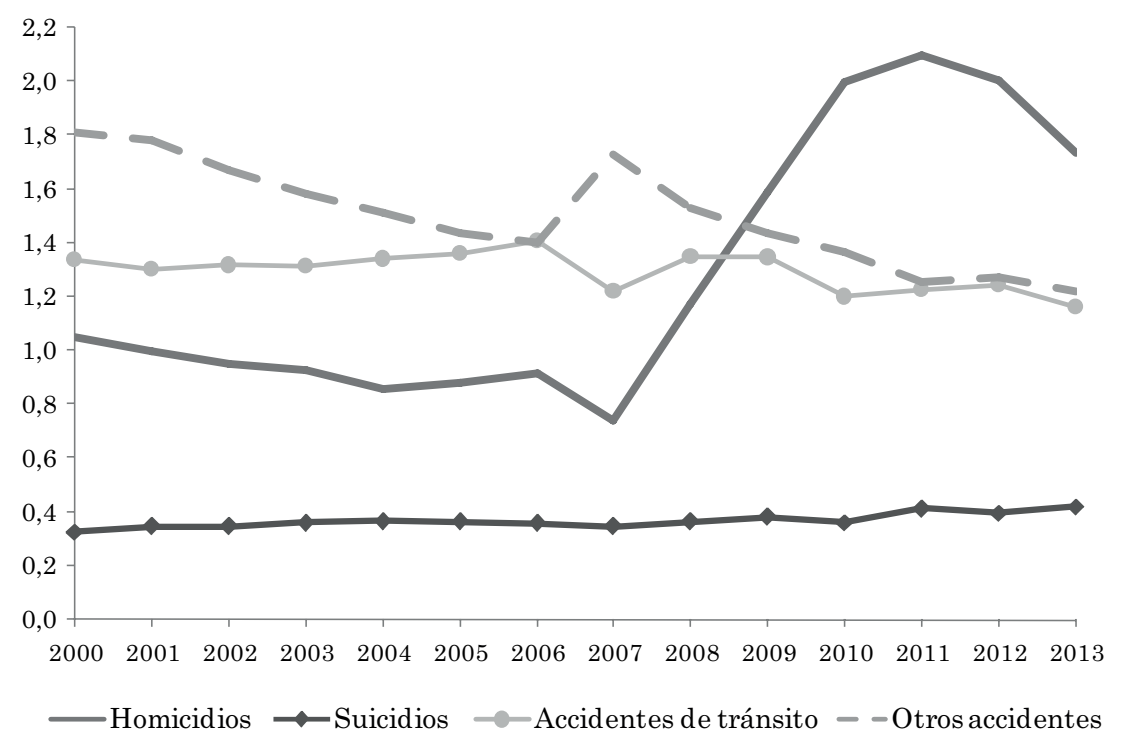

Figura 1. Años de vida perdidos por muertes por causas externas en hombres. México, 2000-2013.

Fuente: Elaboración propia con base en datos de estadísticas vitales de mortalidad $^{(12)}$. 


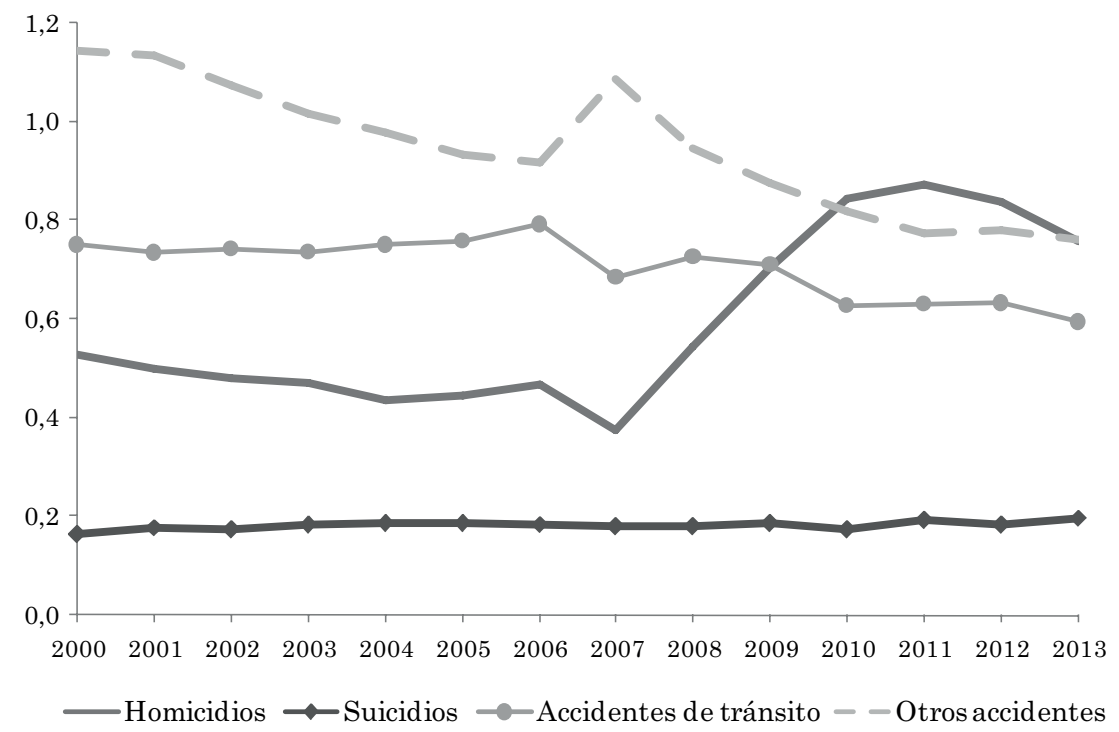

Figura 2. Años de vida perdidos por muertes por causas externas en mujeres. México, 2000-2013.

Fuente: Elaboración propia con base en datos de estadísticas vitales de mortalidad ${ }^{(12)}$.

mostraron una tendencia creciente en el periodo de estudio, al pasar de 0,16 AVP en 2000 a 0,20 AVP en 2013 (con un incremento del $20,1 \%$ ) y, de forma similar a los homicidios, el grupo de edad con los mayores AVP fue el de 15 a 49 años con poco más del $60 \%$ de los AVP por suicidios. Destaca que entre los 15 y los 49 años, los AVP por causas externas de muerte fueron entre 2 y hasta 3,3 veces mayores para hombres que para mujeres. $\mathrm{Al}$ igual que en hombres, los AVP por lesiones de intención no determinada tuvieron, en mujeres, alrededor de 0,14 años en todo el periodo de estudio pero, en 2011, también presentaron un importante incremento (alcanzando 0,19 años).

\section{Contribución al cambio en esperanza de vida}

La esperanza de vida presentó un incremento de 1,3 años entre 2000 y 2013 para ambos sexos: aumentó 1,2 años para hombres (de 70,5 a 71,7 años) mientras que, para mujeres, el cambio fue de 1,3 años (de
76,1 a 77,4 años). Los grupos de edad que más contribuyeron a este cambio fueron el de 0 a 4 años $(0,79$ y 0,62 años para hombres y mujeres respectivamente) y el de 50 a 64 años $(0,25$ años en hombres y 0,23 años para mujeres). En cambio, la mortalidad entre los 15 y los 29 años presentó un incremento que contribuyó negativamente a la esperanza de vida, en 0,11 años para hombres y en 0,01 años en mujeres.

Según causas de muerte, en hombres se destaca un aumento en la esperanza de vida (o una disminución de la mortalidad) por otros accidentes (0,37 años) ocurridas principalmente entre los 15 y los 49 años de edad, y por accidentes de tránsito $(0,11$ años), entre los 30 y los 64 años (Cuadro 1). En cambio, para homicidios y suicidios se presentó un aumento de la mortalidad, con una disminución de la esperanza de vida de 0,42 años y de 0,06 años respectivamente. Estos cambios se centraron entre los 15 y los 49 años de edad para ambas causas de muerte (Figura 3), lo que implica que el aumento de la esperanza de vida, producto de la disminución de la mortalidad por accidentes de 


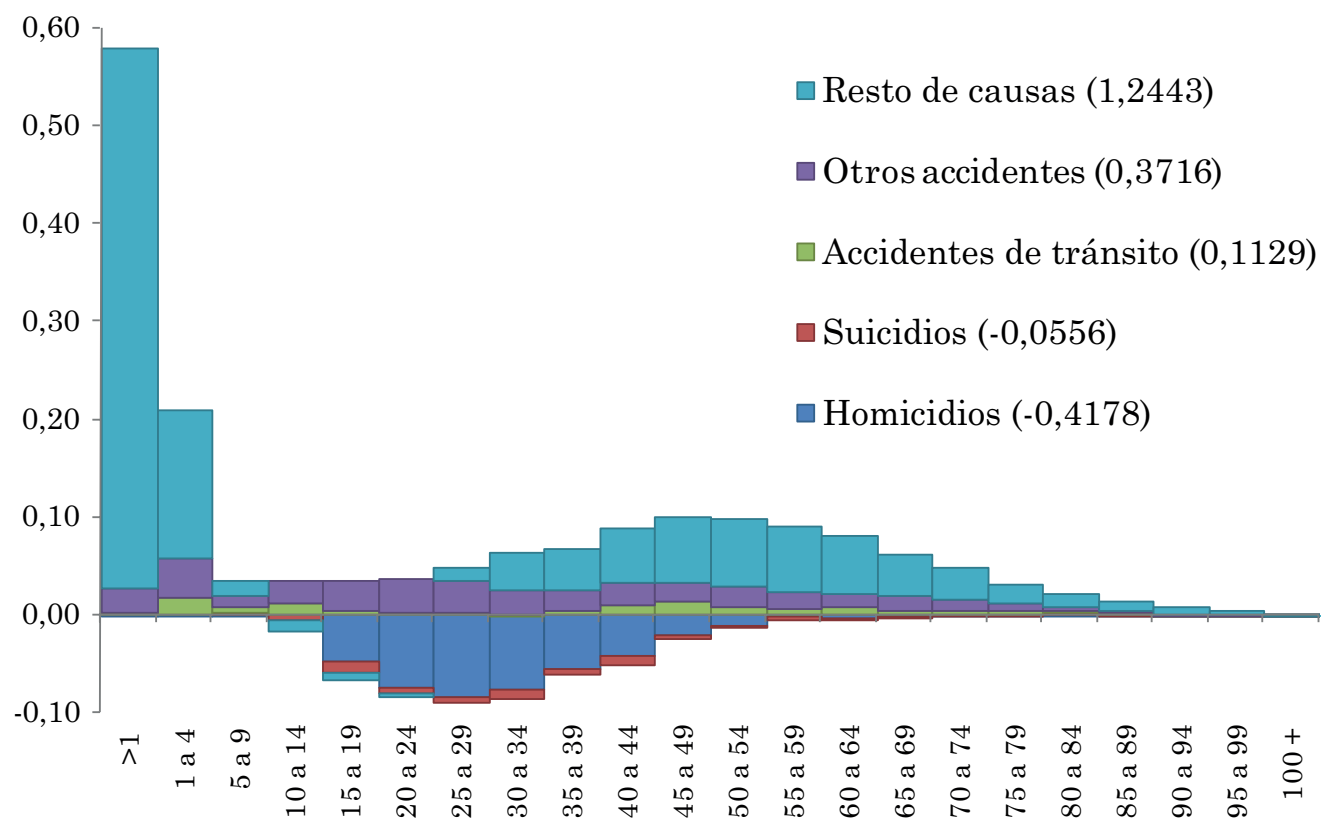

Figura 3. Contribución de las causas externas de muerte al cambio en la esperanza de vida en hombres. México, 2000-2013.

Fuente: Elaboración propia con base en datos de estadísticas vitales de mortalidad $^{(12)}$.

Cuadro 1. Contribución al cambio en la esperanza de vida de las muertes por causas externas, según sexo. México 2000-2013.

\begin{tabular}{|c|c|c|c|c|c|c|c|}
\hline Sexo & Edad & Homicidios & Suicidios & $\begin{array}{l}\text { Accidentes } \\
\text { de tránsito }\end{array}$ & $\begin{array}{c}\text { Otros } \\
\text { accidentes }\end{array}$ & $\begin{array}{l}\text { Resto de } \\
\text { causas }\end{array}$ & Total \\
\hline \multirow{7}{*}{ Hombres } & 0 a 4 & 0,0023 & 0,0000 & 0,0165 & 0,0660 & 0,7053 & 0,7901 \\
\hline & 5 a 14 & 0,0021 & $-0,0044$ & 0,0185 & 0,0325 & 0,0034 & 0,0522 \\
\hline & 15 a 29 & $-0,2077$ & $-0,0210$ & 0,0078 & 0,0990 & 0,0033 & $-0,1186$ \\
\hline & 30 a 49 & $-0,1963$ & $-0,0261$ & 0,0282 & 0,0857 & 0,2080 & 0,0997 \\
\hline & 50 a 64 & $-0,0168$ & $-0,0038$ & 0,0233 & 0,0500 & 0,1969 & 0,2496 \\
\hline & 65 a 84 & $-0,0016$ & $-0,0001$ & 0,0152 & 0,0355 & 0,1081 & 0,1571 \\
\hline & $85+$ & 0,0001 & $-0,0004$ & 0,0035 & 0,0029 & 0,0193 & 0,0254 \\
\hline \multirow{7}{*}{ Mujeres } & 0 a 4 & 0,0001 & 0,0000 & 0,0063 & 0,0426 & 0,5686 & 0,6176 \\
\hline & 5 a 14 & $-0,0011$ & $-0,0018$ & 0,0064 & 0,0107 & 0,0112 & 0,0253 \\
\hline & 15 a 29 & $-0,0329$ & $-0,0146$ & $-0,0019$ & 0,0113 & 0,0331 & $-0,0051$ \\
\hline & 30 a 49 & $-0,0231$ & $-0,0076$ & 0,0099 & 0,0184 & 0,1961 & 0,1936 \\
\hline & 50 a 64 & $-0,0015$ & $-0,0026$ & 0,0094 & 0,0113 & 0,2145 & 0,2312 \\
\hline & 65 a 84 & $-0,0007$ & $-0,0001$ & 0,0073 & 0,0044 & 0,1636 & 0,1746 \\
\hline & $85+$ & 0,0006 & 0,0001 & 0,0020 & 0,0007 & 0,0286 & 0,0320 \\
\hline
\end{tabular}


$\square$ Resto de causas $(1,2156)$

$\square$ Otros accidentes $(0,0995)$

$\square$ Accidentes de tránsito $(0,0393)$

$\square$ Suicidios $(-0,0266)$

$\square$ Homicidios $(-0,0587)$

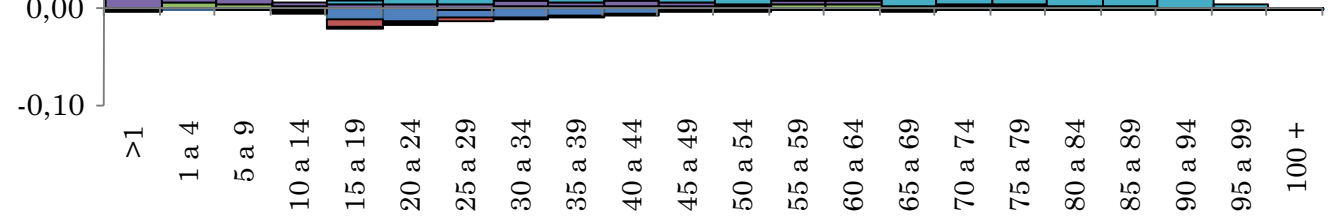

Figura 4. Contribución de las causas externas de muerte al cambio en la esperanza de vida, en mujeres. México, 2000-2013.

Fuente: Elaboración propia con base en datos de estadísticas vitales de mortalidad $^{(12)}$.

tránsito y otros accidentes, quede anulado por el incremento de la mortalidad por homicidios y suicidios. Es importante aclarar que el incremento de la esperanza de vida masculina se centró, entonces, en el resto de las causas de muerte no consideradas en este estudio (1,24 años), principalmente entre 0 a 4 años de edad (0,71 años) y 30 a 49 años $(0,21$ años).

Para la esperanza de vida femenina, la situación es similar, ya que tanto los otros accidentes como los accidentes de tránsito aportaron positivamente $(0,1$ años y 0,04 años respectivamente) aunque, a diferencia de los hombres, estas ganancias se presentaron en casi todos los grupos de edad (de 0 a 64 años para otros accidentes y de 0 a 14 años y 30 a 84 años para accidentes de tránsito) (Cuadro 1). Para las otras dos causas externas de muerte se presentó un aumento de la mortalidad (0,06 años en homicidios y 0,03 años para suicidios), centrado entre los 15 y los 49 años en homicidios y entre los 15 y los 29 años para suicidios (Figura 4). Las ganancias por accidentes de tránsito y otros accidentes fueron prácticamente anuladas por el aumento de la mortalidad de homicidios y suicidios, lo que implica que el cambio en la esperanza de vida femenina se dio por otras causas de muerte, incluidas en el resto de las causas $(1,22$ años), principalmente en el grupo de 0 a 4 años de edad (0,57 años) y el de 50 a 64 años de edad (0,21 años).

\section{DISCUSIÓN}

La mortalidad por causas externas es uno de los principales problemas de salud pública en Latinoamérica, y puede ser considerada como indicador de carencia, tanto en el acceso y la calidad de la atención médica, como de mecanismos de control y prevención que son responsabilidad de la población y del Estado y que podrían reducir la exposición al riesgo de accidentes y agresiones ${ }^{(23)}$. 
Los resultados de este estudio permiten corroborar el gran impacto que tiene la mortalidad por causas externas en la salud de la población ${ }^{(17,24,25)}$, a la vez que dan cuenta de su comportamiento diferencial por causa de muerte, edad y sexo. En su mayoría, las defunciones se presentan en hombres jóvenes y adultos de 15 a 49 años de edad ${ }^{(11,26)}$. La mortalidad por causas externas en hombres permaneció relativamente constante y ha disminuido ligeramente en mujeres. Según causa de muerte, en el año 2000, los accidentes de tránsito y otros accidentes provocaban los mayores AVP, tanto en hombres como en mujeres. Sin embargo, la mortalidad por estas causas ha disminuido hasta 2013, como se ha visto en otras investigaciones $s^{(1,8)}$. Esta disminución de la mortalidad se reflejó también en el aporte positivo de estas causas de muerte a la esperanza de vida. Se presentó un incremento significativo de la mortalidad por homicidios a partir de 2008, y un paulatino aumento de los suicidios, el cual ha continuado desde hace más de 40 años $^{(14,27)}$, lo que propició que el aporte positivo de los accidentes de tránsito y otros accidentes a la esperanza de vida haya sido prácticamente cancelado por el incremento de la mortalidad por homicidios y suicidios. Es importante resaltar que, en 2011 -año con la mayor mortalidad por homicidios en la historia reciente del país-, se presentó un incremento inusual de las lesiones de intención no determinada, lo que puede ser indicativo de cierta subestimación del impacto de la mortalidad por las causas externas aquí consideradas (y, en particular, de los homicidios) en la esperanza de vida del país; situación que se recomienda abordar más a fondo en investigaciones futuras sobre el tema.

Según sexo, se corroboró que los varones presentan una mayor mortalidad por causas externas que las mujeres, como en otras investigaciones sobre el tema ${ }^{(6,11,24)}$. Se observó también que el mayor impacto de la mortalidad por estas causas, para hombres, así como los mayores cambios en la esperanza de vida, se dieron entre los 15 y los 49 años. En mujeres, el impacto de la mortalidad por accidentes de tránsito y otros accidentes se distribuyó entre diferentes grupos de edad mientras que, por homicidios y suicidios, la mayor mortalidad y las principales disminuciones en la esperanza de vida se dieron entre los 15 y los 49 años. Esta concentración de la mortalidad por causas externas en los adolescentes, jóvenes y adultos jóvenes, especialmente en hombres, es uno de los factores que determina que este tipo de causas de muerte sean una de las principales fuentes de AVP en la población mexicana ${ }^{(1,11,24)}$.

Esto es relevante, dado que las muertes por causas externas, además de trágicas pérdidas humanas ${ }^{(3)}$, tienen altos costos económicos y sociales $^{(1)}$; ocasionan daño físico, discapacidad y/o secuelas psicológicas, un gran número de $\mathrm{AVP}$, una disminución de la esperanza de vida ${ }^{(25)}$, y es una forma de expresión del deterioro de la calidad de vida de la población ${ }^{(24)}$. El estudio de la violencia desde una perspectiva de salud pública permite realizar un análisis integral de sus factores asociados, así como de los efectos negativos que tiene en la sociedad y la salud de la población ${ }^{(28,29,30)}$. En este aspecto, la mortalidad por causas externas y, en particular, las intencionales (homicidios y suicidios) se ha relacionado con factores estructurales como condiciones socioeconómicas desfavorables, que propician la desigualdad social, eco-

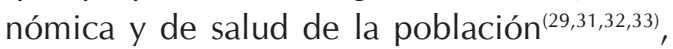
o con la cohesión y el capital social ${ }^{(34,35)}$. En México, otros factores altamente relacionados con estas causas son los altos índices de impunidad, que forman parte de un proceso permanente que ha permeado toda la sociedad mexicana y refleja una creciente incapacidad del Estado para hacer efectivas sus propias normas ${ }^{(36,37)}$. La probabilidad de que una persona que haya cometido un crimen sea aprehendida es muy baja, al igual que la de recibir sentencia, o de cumplirla si la recibe ${ }^{(38)}$, lo que se refleja en que el $93 \%$ de los delitos quedan impunes y casi el 99\% no recibe ningún tipo de sanción ${ }^{(36)}$.

Paralelamente, se corroboró que los hombres tienen hasta tres veces más riesgo de morir por causas externas que las mujeres, principalmente, los adolescentes y adultos jóvenes ${ }^{(39)}$. La mortalidad diferencial por este 
tipo de causas entre hombres y mujeres, en muchos casos, no se explica por diferencias fisiológicas sino que está asociada a conductas de dichos sujetos, provenientes de un aprendizaje social diferenciado que ocurre en el proceso de construcción de sus identidades de género ${ }^{(40,41)}$. Se argumenta que la dinámica diferencial de la morbimortalidad por este tipo de causas entre hombres y mujeres es un reflejo de los patrones masculinos de comportamiento, de los procesos de socialización, de los modelos de identidad masculina y de los roles que la sociedad les asigna $^{(42)}$. En este sentido, la masculinidad a la cual se hace referencia comprende comportamientos esperados (como competencia, riesgo, control y aventura) frente a las distintas situaciones a las que los hombres se enfrentan diariamente. Como hombres, los niños aprenden a correr riesgos e internalizar los comportamientos asociados con la masculinidad $^{(42)}$, lo que se expresa mayormente durante la adolescencia y la adultez joven, edades en las cuales la exposición intencional a situaciones de riesgo se convierte en una situación social esperada que los legitima como varones aunque, en el proceso, arriesguen su salud y bienestar $y$, en algunos casos, los lleve a encontrar su propia muerte ${ }^{(43,44)}$. Por lo tanto, muchos de los fallecimientos por causas externas pueden surgir de una exposición intencional a situaciones de riesgo derivada de conductas relacionadas con la masculinidad. Por todo ello, los principales factores asociados con la mortalidad por causas externas son el género y la edad, es decir que el principal factor demográfico asociado con este fenómeno es el hecho mismo de ser hombre ${ }^{(42,43)} y$, particularmente, hombre joven.

Otro aspecto relevante, asociado con la violencia y los accidentes, es el abuso de sustancias como las drogas y el alcohol ${ }^{(45,46)}$, el cual es mayor en hombres, por lo que los expone a un mayor riesgo de fallecer por estas causas ${ }^{(47)}$. El predominio masculino en el consumo de alcohol y drogas se ha relacionado con un proceso de aprendizaje social mediante el cual, a partir del abuso de sustancias, legitiman su exposición deliberada ante escenarios de riesgo asociados con conductas masculinas esperadas ${ }^{(42,47)}$.

Por otro lado, si bien el número absoluto de muertes por accidentes de tránsito se incrementó en los últimos años ${ }^{(8)}$, la situación cambia al analizar su impacto en la esperanza de vida y el número de AVP, ya que existió una disminución de la mortalidad por esta causa en la población mexicana entre 2000 y 2013. La mortalidad por accidentes de tránsito se concentra en adultos jóvenes, principalmente en hombres ${ }^{(8,48)}$, y los más afectados son los peatones, seguidos por los motociclistas y ciclistas, es decir, usuarios vulnerables ${ }^{(48)}$. Uno de los principales factores de riesgo asociados con accidentes de tránsito es el aumento del número de vehículos en circulación ${ }^{(8)}$, así como las conductas de riesgo como exceso de velocidad, abuso de sustancias (alcohol o drogas), falta de uso del cinturón de seguridad, no respetar señalamientos de tránsito, y el uso de distractores (como teléfonos móviles) mientras se maneja, entre muchos otros ${ }^{(39,43)}$.

Para homicidios, la tendencia reciente mostró un incremento significativo de la mortalidad a partir de 2008, con una mayor prevalencia en hombres entre los 15 y los 49 años de edad. Esto ha sido asociado, a nivel micro, con cuestiones de género ${ }^{(49)} y$, a nivel macro, con las condiciones sociales, económicas y políticas del país -tales como el atraso económico, la pobreza, la desigualdad social, la exclusión social, educativa y laboral-; la distribución arbitraria y desigual de la riqueza, la cual genera inequidades y marginalidad ${ }^{(50)}$; la debilidad del Estado, la impunidad y la corrupción; la dificultad de los jóvenes para conseguir trabajo; la disponibilidad de armas de fuego ${ }^{(51)}$; la ruptura de controles sociales tradicionales ejercidos a través de la familia y la escuela; la rápida urbanización sin planificación; las nuevas condiciones de vida; los acelerados cambios demográficos; y las estrategias gubernamentales frente al crimen organizado ${ }^{(52)}$.

Por último, la mortalidad por suicidios continuó con la tendencia creciente reportada desde la década de 1970, revelando importantes diferencias por edad y sexo $^{(12)}$. 
Este incremento, junto con la disparidad entre hombres y mujeres, se ha asociado con factores que varían de acuerdo al grupo de edad y, en algunos casos, al sexo ${ }^{(53,54)}$. Entre los jóvenes, los principales factores son la depresión y otros desórdenes de comportamiento ${ }^{(49)}$, y el aumento del consumo de drogas y alcohol. Entre los adultos mayores, se encuentran trastornos depresivos asociados con la discapacidad y el deterioro inherente al proceso de envejecimiento -especialmente en hombresjunto con el aislamiento y la pérdida de autoestima derivados del retiro de actividades consideradas socialmente productivas ${ }^{(55)} \mathrm{y}$, en ambos sexos, con el incremento de enfermedades crónicodegenerativas, las cuales pueden provocar gran dolor en quienes las padecen $^{(54)}$. Un aspecto adicional, que explica la diferencia de mortalidad por suicidios entre sexos, se basa en la selección del medio para producir la muerte, ya que los hombres utilizan con más frecuencia medios más letales ${ }^{(56)}$ a pesar de que la ideación suicida y los intentos son más frecuentes en las mujeres ${ }^{(25)}$.

La mortalidad por causas externas, en su mayoría, se puede prevenir por medio de intervenciones diseñadas para modificarla y disminuir sus efectos. Para ello, es necesario obtener tantos conocimientos como sea posible ${ }^{(5)}$ sobre los subgrupos de la población en los que tiene mayor impacto para delimitar grupos en alto riesgo ${ }^{(25)}$, sobre los factores asociados que intervienen en este tipo de muertes y los cambios ocurridos en el tiempo; todo ello encaminado al diseño, ejecución, vigilancia y evaluación de intervenciones ${ }^{(5)}$, programas y estrategias de prevención y tratamiento oportuno ${ }^{(6)}$. Los programas de prevención deben tomar en cuenta que se puede disminuir el número de accidentes de tránsito por medio de restricciones en el uso de bebidas alcohólicas, reducción de límites de velocidad, uso obligatorio del cinturón de seguridad y el respeto a las señales de tránsito ${ }^{(48)}$; de suicidios, a través de la detección y tratamiento oportuno de las causas psicopatológicas relacionadas con esta causa; y de homicidios, por medio de planes de prevención de la violencia ${ }^{(17)}$.
Dado que se trata de un fenómeno multicausal, las acciones encaminadas a prevenir la violencia, los accidentes y sus consecuencias se deben generar tomando en cuenta los grupos de edad en los que tienen mayor impacto (adolescentes y adultos jóvenes), con perspectiva de género ${ }^{(42,43)}$, e incluyendo la participación de "todos los sectores de la sociedad, con un enfoque multidisciplinario e intersectorial"(1 p.104). A partir de los resultados de este trabajo y tomando en cuenta la importancia que han tenido las muertes por causas externas en el perfil epidemiológico de México, se hace necesario contar con información precisa que permita vigilar la incidencia de este tipo de causas de muerte $y$, paralelamente, desarrollar estudios multidisciplinarios que se concentren en la dinámica de los múltiples factores asociados con la mortalidad por este tipo de causas.

\section{Limitaciones}

Actualmente, se dispone de poca información sobre la violencia como fenómeno social $^{(13)}$. En esta investigación, la información se centró en los datos de mortalidad, dado que se registran y están disponibles de manera más amplia ${ }^{(5)}$. Sin embargo, es importante señalar que el sistema de información de las estadísticas vitales de mortalidad puede presentar un subregistro significativo de las muertes ocurridas por hechos violentos y por accidentes, ya sea porque las muertes provocadas durante enfrentamientos armados $\mathrm{u}$ homicidios a manos de grupos ilegales podrían no estar contabilizadas por diversas circunstancias, quedando fuera del alcance de los registros nacionales de mortalidad; o, en el caso de los suicidios, por el estigma social asociado con esta causa de muerte o por la renuencia a clasificarlo como tal si las circunstancias que lo rodean no son del todo claras ${ }^{(57)}$. Aunado a ello, las estadísticas vitales tienen además otro tipo de limitaciones, como la falta de uniformidad en las definiciones del registro, una cobertura inadecuada en regiones poco accesibles y, en algunos casos, errores de omisión. 
Una limitación adicional fue que se enfocó en fallecimientos por causas externas sin poder tomar en cuenta los numerosos incidentes no letales que ocurren por cada defunción, ya que estas últimas son solo una pequeña parte del conjunto de la violencia(24) y los accidentes. No toda agresión ocasiona lesiones suficientemente graves, por lo que no existe registro de su ocurrencia $y$, por ende, no se cuenta con estimaciones nacionales e internacionales precisas sobre su prevalencia ${ }^{(5)}$, por lo que es probable que la información utilizada esté subestimada. A pesar de que la mortalidad por causas externas es solo la parte más visible de un problema mayor que tiene múltiples raíces y graves consecuencias, las conclusiones que se pueden sacar a partir de las cifras oficiales son esencialmente correctas ${ }^{(58)}$.

\section{REFERENCIAS BIBLIOGRÁFICAS}

1. Yunes J, Zubarew T. Mortalidad por causas violentas en adolescentes y jóvenes: un desafío para la región de las Américas. Revista Brasileira de Epidemiologia. 1999;2(3):45-57.

2. Cardona D, Peláez E, Aidar T, Ribotta B, Alvarez MF. Mortalidad por causas externas en tres ciudades latinoamericanas: Córdoba (Argentina), Campinas (Brasil) y Medellín (Colombia), 1980-2005. Revista Brasileira de Estudos de População. 2008;25(2):335-352.

3. Sánchez R, TejadaP, MartínezJ. Comportamiento de las muertes violentas en Bogotá, 1997-2003. Revista de Salud Pública. 2005;7(3):254-267.

4. Organización Panamericana de la Salud. La salud en las Américas. Washington DC: OPS; 1998.

5. Organización Mundial de la Salud. Informe mundial sobre la violencia y la salud. Ginebra: OMS; 2003.

6. Burrone MS, Bella M, Acosta L, Villace B, López de Neira MJ, Fernández R, Enders JE. Estudio de muertes por causas violentas: un análisis de tendencia en jóvenes, Argentina, 2000-2008. Cadernos Saúde Coletiva. 2012;20(4):460-465.

7. Organización Mundial de la Salud. Global status report on violence prevention 2014. Ginebra: OMS; 2014

8. Consejo Nacional para la Prevención de Accidentes. Tercer informe sobre la situación de la seguridad vial. México DF: Secretaría de Salud; 2013.

9. World Health Organization. Global Health Observatory: The data repository [Internet]. c2016 [citado 10 sep 2015]. Disponible en: http://www. who.int/gho/database/en
10. Dávila-Cervantes CA, Pardo-Montaño AM. Análisis de la tendencia de la mortalidad por homicidios en México entre 2000 y 2010. Revista Gerencia y Políticas de Salud. 2013;12(24):163-183.

11. Canudas-Romo V, García-Guerrero VM, Echarri-Cánovas CJ. The stagnation of the Mexican male life expectancy in the first decade of the $21 \mathrm{st}$ century: the impact of homicides and diabetes mellitus. Journal of Epidemiology of Community Health. 2015;69(1):28-34.

12. Instituto Nacional de Estadística y Geografía. Estadísticas vitales de mortalidad [Internet]. INEGI [citado 10 sep 2015]. Disponible en: http://goo.gl/ $\mathrm{KO} 6 \mathrm{QCr}$

13. Escalante F. Panorama del homicidio en México. Esquema de análisis territorial 1990-2007. En: Alvarado A, Serrano M, (ed). Capítulo XV: Seguridad nacional y seguridad interior: Los grandes problemas de México. México: El Colegio de México; 2010. p. 301-330.

14. Martínez C. Población y salud mental en México: Reflexiones y un ejercicio de aproximación mediante las variaciones de la mortalidad por suicidio. Estudios Demográficos y Urbanos. 2010;25(3):663-712.

15. Instituto Nacional de Estadística y Geografía. Los adultos mayores en México: Perfil sociodemográfico al inicio del siglo XXI. Aguascalientes: Instituto Nacional de Estadística y Geografía; 2005.

16. Rockett IR, Regier MD, Kapusta ND, Coben JH, Miller TR, Hanzlick RL, et al. Leading causes of unintentional and intentional injury mortality: United States, 2000-2009. American Journal of Public Health. 2012;102(11):e84-92.

17. Serfaty EM, Foglia VL, Masaútis AE, Negri GM. Mortalidad por causas violentas en Argentina 1991-2000. Vertex. 2003;14(Supl 2):S40-S48. 
18. Arriaga E. Los años de vida perdidos: Su utilización para medir el nivel y cambio de la mortalidad. Notas de Población. 1996;24(63):7-38.

19. Bocco M. La relación entre los años de vida perdidos y la esperanza de vida: aplicaciones para el análisis de la mortalidad. Notas de Población. 1996;24(63):39-60.

20. Andrevv EM, Shkolnikov VM, Begun AZ. Algorithm for decomposition of differentials between aggregate demographic measures and its application to life expectancies, healthy life expectancies, parity progression ratios and total fertility rates. Demographic Research. 2002;7(14):499-522.

21. Partida-Bush V. Cambios en los mercados laborales de México de 2000 a 2010 mediante esperanzas de vida activa. Papeles de Población. 2014;20(81):121-164.

22. Gayet C, Partida-Bush V, Dávila-Cervantes CA. Mortalidad por VIH/SIDA en México: Un aporte demográfico. Papeles de Población. 2014;20(79):9-38.

23. Gómez RD. La mortalidad evitable como indicador de desempeño de la política sanitaria: Colombia 1985-2001. Medellín: Universidad de Antioquia; 2008.

24. Lodoño JL, Guerrero R. Violencia en América Latina: epidemiología y costos. New York: Banco Interamericano de Desarrollo; 1999.

25. Híjar-Medina M, López-López MV, BlancoMuñoz J. La violencia y sus repercusiones en la salud: reflexiones teóricas y magnitud del problema en México. Salud Pública de México. 1997;39:565-572.

26. Briceño-León R. La comprensión de los homicidios en América Latina: ¿Pobreza o institucionalidad? Ciência \& Saúde Coletiva. 2012;17(12):3159-3170

27. Hernández-Bringas HH, Flores-Arenales R. El suicidio en México. Papeles de Población. 2011;17(68):69-101

28. Soberón G. La violencia como un grave problema de salud pública: Caleidoscopio de la salud. México DF: Fundación Mexicana para la Salud; 2002.

29. Briceño-León R, Villaveces A, ConchaEastman A. Understanding the uneven distribution of the incidence of homicide in Latin America. International Journal of Epidemiology. 2008;37(4):751-757.
30. Lee BX, Marotta PL, Blay-Tofey M, Wang W, Bourmonta S. Economic correlates of violent death rates in forty countries 1962-2008: A cross-typological analysis. Aggression and Violent Behavior. 2014;19(6):729-737.

31. Santos SM, Barcellos C, Sa Carvalho M. Ecological analysis of the distribution and socio-spatial context of homicides in Porto Alegre, Brazil. Health and Place. 2006;12(1):38-47.

32. Briceño-León R. Violencia interpersonal: salud pública y gobernabilidad. En: Minayo MCS, Coimbra Jr CE, (comp). Críticas e atuantes. Rio de Janeiro: Editora Fiocruz; 2005. P. 649-663.

33. Spinelli H, Alazraqui M, Macías G, Zunino G, Nadalich JC. Muertes violentas en la Ciudad Autónoma de Buenos Aires. Buenos Aires: OPS; 2005.

34. Kawachi I, Kennedy BP, Wilkinson RG. Crime: social disorganization and relative deprivation. Social Sciences and Medicine. 1999;48(6):719-731.

35. Minamisava R, Nouer SS, Morais-Neto OL, Melo LK, Andrade AL. Spatial clusters of violent deaths in a newly urbanized region of Brazil: highlighting the social disparities. International Journal of Health Geographics. 2009;8:66. doi: 10.1186/1476-072X-8-66

36. Menéndez E. Violencias en México: las explicaciones y las ausencias. Alteridades. 2012;22(43):177-192.

37. O'Donnell G. Acerca del Estado, la democratización y algunos problemas conceptuales: Una perspectiva latinoamericana con referencias a países poscomunistas. Desarrollo Económico. 1993;33(130):1-32.

38. Azaola E. Crimen, castigo y violencias en México. Quito: Facultad Latinoamericana de Ciencias Sociales; 2008.

39. Organización Mundial de Salud. Declaración de los jóvenes sobre la seguridad vial. Ginebra: OMS; 2007.

40. Figueroa JG. El ser hombre desde el cuidado de sí: algunas reflexiones. VI Seminario Internacional sobre Familia; 21-24 abr 2015; Manizales, Colombia.

41. Burin M, Meler I. La masculinidad: Diversidad y similitudes entre los grupos humanos. En: Burin $M$, Meler I, (ed). Varones: género y subjetividad masculina. Buenos Aires: Paidós; 2000. p. 71-121. 
42. Figueroa JG. El derecho a la salud en la experiencia de los varones: ¿un concepto ambivalente en los modelos de masculinidad vigentes? Coeducando. 2007;1;77-97.

43. Treviño-Siller S, Villanueva-Borbolla $M$, Marcelino-Sandoval Y, Álvarez-Guillén F. Masculinidad, accidentes viales y políticas públicas. En: Figueroa JG, (ed). Políticas públicas y la experiencia de ser hombre: Paternidad, espacios laborales, salud y educación. México DF: El Colegio de México; 2014.

44. Mansfield A, Dais M, Mahalik J. Why won't he go to the Doctor?: The psychology of men's help seeking. International Journal of Men's Health. 2003;2(2):93-109.

45. Rivara F, Mueller BA, Somes G, Mendoza CT, Rushford NB, Kellerman AL. Alcohol and illicit drug abuse and the risk of violent death in the home. JAMA. 1997;278:569-575.

46. Gray D, Coon H, McGlade E, Callor W, Byrd J, Viskochil J, et al. Comparative analysis of suicide, accidental, and undetermined cause of death classification. Suicide \& Life-Threatening Behavior. 2014;44(3):304-316.

47. Goldstein PJ. The drugs/violence nexus: a tripartite conceptual framework. Journal of Drug Issues. 1985;15(4):493-506.

48. World Health Organization. Global status report on road safety 2013. Geneva: Who; 2013.

49. Sheehan CM, Rogers RG, Williams GW, Boardman JD. Gender differences in the presence of drugs in violent deaths. Addiction. 2013;108(3):547-555

50. Cardona M, García HI, Giraldo CA, López MV, Suárez CM, Corcho DC, et al. Homicides in
Medellín, Colombia, from 1990 to 2002: victims, motives and circumstances. Cadernos de Saúde Pública. 2005;21(3):840-51.

51. González-Pérez GJ, Vega-López MG, VegaLópez A, Muñoz-de-La-Torre A, Cabrera-Pivaral CE. Homicidios en la adolescencia en México, 1979-2005: evolución y variaciones sociogeográficas. Papeles de Población. 2009;15(62):109-141.

52. Escalante F. Homicidios 2008-2009: La muerte tiene permiso. Nexos. 2011;397:36-49.

53. Gunnell D. Reporting suicide: the effect of media coverage on patterns of self harm. BMJ. 1994;308:1446.

54. Rojas-Cabrera ES. Mortalidad por causas violentas en adolescentes y jóvenes de dos ciudades del Cono Sur: Córdoba (Argentina) y Porto Alegre (Brasil), 1990-2010. Ciência \& Saúde Coletiva. 2015;20(1):29-37.

55. Kerkhof AJ, Visser AP, Diekstra RF, Hirschorn PM. The prevention of suicide among older people in The Netherlands: Interventions in community mental health care. Crisis 1991;12(2):59-72.

56. Taylor CM, Wicks WJ. The choice of weapons: A study of methods of suicide by sex, race and region. Suicide \& Life-Threatening Behavior. 1980;10:142-149.

57. Timmermans S. Suicide determination and the professional authority of medical examiners. American Sociological Review. 2005;70(2):311-333.

58. Speechley M, Staravsky KM. The adequacy of suicide statistics for use in epidemiology and public health. Canadian Journal of Public Health. 1991;82(1):38-42.

\section{FORMA DE CITAR}

Dávila Cervantes CA, Pardo Montaño AM. Análisis de la tendencia e impacto de la mortalidad por causas externas: México, 2000-2013. Salud Colectiva. 2016;12(2):251-264. doi: 10.18294/sc.2016.743.

Recibido: 7 de octubre de 2015 | Versión final: 8 de marzo de 2016 | Aprobado: 4 de abril de 2016

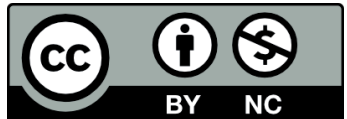

Este obra está bajo una licencia de Creative Commons Reconocimiento-NoComercial 4.0 Internacional. Reconocimiento - Permite copiar, distribuir y comunicar públicamente la obra. A cambio, se debe reconocer y citar al autor original. No Comercial - Esta obra no puede ser utilizada con finalidades comerciales, a menos que se obtenga el permiso.

http://dx.doi.org/10.18294/sc.2016.743 